\title{
Use of the GATE Monte Carlo package for dosimetry applications
}

\author{
D. Visvikis, ${ }^{a^{*}}$ M. Bardies, ${ }^{b}$, S. Chiavassa, ${ }^{b}$, C. Danford, ${ }^{\text {c }}$, A. Kirov, ${ }^{c}$, F. Lamare, ${ }^{a}$, L. \\ Maigne, ${ }^{\mathrm{d}}$, S. Staelens, ${ }^{\mathrm{e}}$, R. Taschereau, \\ ${ }^{a}$ INSERM U650, LaTIM, University Hospital Medical School, F-29609, Brest, France \\ ${ }^{b}$ INSERM U601, CHU Nantes, F-44093, Nantes, France \\ ${ }^{c}$ Department of Medical Physics, MSKCC, New York, USA \\ ${ }^{d}$ Departement de Curietherapie-Radiotherapie, Centre Jean Perrin, F-63000, Clemont-Ferrand, France \\ ${ }^{e}$ UGent-ELIS, St-Pietersnieuwstraat, 41, B-9000 Gent, Belgium \\ ${ }^{f}$ CRUMP Institute for Molecular Imaging, UCLA, Los Angeles, USA
}

\begin{abstract}
One of the roles for MC simulation studies is in the area of dosimetry. A number of different codes dedicated to dosimetry applications are available and widely used today, such as MCNP, EGSnrc and PTRAN. However, such codes do not easily facilitate the description of complicated 3D sources or emission tomography systems and associated data flow, which may be useful in different dosimetry application domains. Such problems can be overcome by the use of specific MC codes such as GATE, which is based on Geant4 libraries, providing a scripting interface with a number of advantages for the simulation of SPECT and PET systems. Despite this potential, its major disadvantage is in terms of efficiency involving long execution times for applications such as dosimetry. The strong points and disadvantages of GATE in comparison to other dosimetry specific codes are discussed and illustrated in terms of accuracy, efficiency and flexibility. A number of features, such as the use of voxelised and moving sources, as well as developments such as advanced visualisation tools and the development of dose estimation maps allowing GATE to be used for dosimetry applications are presented. In addition, different examples from dosimetry applications with GATE are given. Finally, future directions with respect to the use of GATE for dosimetry applications are outlined.
\end{abstract}

Keywords : Monte Carlo; GATE; dosimetry; efficiency

\footnotetext{
* Corresponding author. Tel.: +33298018114; fax: +33298018124; e-mail: Visvikis.Dimitris@univ-brest.fr.
} 


\section{Introduction}

Since its release as an open source package for Monte Carlo (MC) simulations in May 2004, GATE (GEANT4 Application to Tomographic Emission) ${ }^{1}$, has found a widespread acceptance for the applications it has been originally intended for, such as simulation of human and small animal emission tomography systems. However, as every new tool GATE also offers new functionality which could be beneficial in other related fields. One such potential area is in the field of dosimetry, considering both internal and external radiation therapy applications. A number of different codes dedicated to dosimetry applications are available and widely used today, such as MCNP $^{2}$ and EGSnrc ${ }^{3}$, or radiotherapy dedicated codes such as $\mathrm{XVMC}^{4}$. A brief discussion on the advantages and disadvantages of these codes in comparison to GATE follows in order to identify the potential impact of GATE for dosimetry.

The criteria for comparison of Monte Carlo codes for dosimetry were previously discussed by Rogers and Mohan ${ }^{5}$. In addition to the accuracy and computational efficiency criteria underlined in that paper, availability, convenience of use, flexibility for simulating complex detector geometries and experimental arrangements as well as support and maintenance are also factors affecting the choice of a Monte Carlo code. Prior to the release of GATE, several published reviews summarized the properties and performance of various Monte Carlo codes for emission tomography applications ${ }^{6}$, as well as in photon and electron external radiotherapy ${ }^{7-9}$. A large variety of models, of variable accuracy and completeness, exist in these different codes. A very detailed comparison of the physics models and cross section databases used by the different codes is given by Verhaegen and Seuntjens ${ }^{8}$ and we point the reader to Table 1 in that paper for details. In general, the low energy physics processes affecting dosimetric accuracy are more accurately modeled by EGSnrc, which underwent a very stringent validation to subpercent level of accuracy in modeling detector response for external beam ${ }^{5,10,11}$. EGSnrc has also a better developed electron transport algorithm with improved energy loss evaluation techniques, correct fictitious discrete interactions, well developed variance reduction techniques ${ }^{12}$ and dose statistics tools $^{13}$. Subsequently, considering all these features it is a good choice for an accuracy and computational speed standard. The main reported advantage of MCNP over EGSnrc is the advanced geometry description tools ${ }^{14}$. On the other hand, GATE has certain attractive features for dosimetry calculations. Some of them are inherited from GEANT4 and some are additionally developed. They include a very flexible simulation geometry input capable to accommodate a large variety of detector and source geometries; including a user friendly implemented voxelized source, a virtual clock allowing to simulate temporal phenomena such as source and detector movements and source decay; a large variety of physics models including for example photo-nuclear reactions which do play a role in higher energy external beam dosimetry ${ }^{15}$ and shielding applications $^{16}$. Validation of GEANT4's accuracy for dosimetric calculations has also been performed ${ }^{17-}$ ${ }^{20}$, however electron step artifacts restricting the accuracy of dose simulations in ion chambers have been observed ${ }^{20}$.

The objective of this paper is to describe the modules currently available within GATE allowing dose map calculations and discuss the performance of GATE in terms of accuracy and efficiency. A number of examples are given at the end of the paper on dosimetry applications already performed with GATE. A discussion follows at the end on the future directions and developments for the use of GATE in dosimetry applications.

\section{Materials and Methods}

Firstly, in order to realize the potential of GATE for dosimetry applications a dose map calculation module was developed. This useful feature allows investigators to obtain dose imparted to the subject simultaneously with information regarding the image formation process (PET, CT, SPECT, radiograph). The detailed mechanism of dose collection is described in the following section.

\subsection{Dose map calculation}


Complex phantoms, such as a human torso (from CT) or a realistic mouse, can be described in GATE through the use of voxellized phantoms. Each voxel in the phantom is linked to a material description endowing it with attenuation properties (essentially elemental composition and density), required for proper particle tracking. For visualization purposes, voxels are also assigned color and transparency attributes. Voxellized phantoms are implemented in GATE through the G4PVParameterised class of GEANT4 ${ }^{21}$.

In a given simulation, there can be any number of voxellized phantoms. Dose may be collected in the phantom through the "addDoseOutput" command. At the end of the simulation run, a binary file containing dose deposited in each voxel (in cGy) is written to disk. Optionally, a second file is written which contains the relative uncertainty (fraction between 0 and 1 ) of dose for each voxel.

Phantoms, voxellized or not, are usually declared as "sensitive detector" through the "attachPhantomSD" or "attachVoxelPhantomSD”. In GEANT4 terminology, a sensitive detector is an object, attached to a volume present in the geometry that is notified when interactions occur in that volume. Typically, a sensitive detector will collect information about the interaction such as energy deposit and create an object, called a "hit", representing that interaction. When all particles issued from a single primary emitted particle have been tracked (end of event), collected hits for this event can be inspected and further processed.

GATE uses the sensitive detector mechanism to collect dose information. At the beginning of the simulation run, a matrix having the same dimensions as the phantom is created to accumulate dose. If uncertainty calculations have been requested, two additional matrices (also having the same dimensions) are created, one to count the number of hits and the other to accumulate the sum of the squares of energy deposits. During the simulation run and for every interaction in a phantom, the sensitive detector (GatePhantomSD class of GATE) keeps the energy deposit and spatial coordinates of the interaction in a (GatePhantomHit) object. At the end of each event, hit objects are reviewed and from the (continuous) spatial coordinates of the interaction, (discrete) matrix indices are calculated and dose is accumulated in the dose matrix at those coordinates. Dose is calculated by dividing the energy deposit by the mass of the voxel (volume times density obtained from the material definition). Optionally, the count and sum-of-squares matrices are also updated.

At the end of the run, the dose matrix and optionally the uncertainty matrix are written as binary files. Relative uncertainty is calculated as $\hat{d} / d$, where $d=\sigma / \sqrt{N}$ is the standard error of the mean calculated with the unbiased variance estimator:

$$
\sigma^{2}=\frac{1}{N(N-1)}\left[N \sum_{i} d_{i}^{2}-\left(\sum_{i} d_{i}\right)^{2}\right]
$$

The final formula involves all three matrices: dose, count and sum-of-squares:

$$
\frac{\hat{d}}{d}=\left[\frac{N}{N-1}\left(\frac{\sum_{i} d_{i}^{2}}{\left(\sum_{i} d_{i}\right)^{2}}-1\right)\right]^{1 / 2}
$$

\subsection{Dosimetry Benchmark}

GATE comes with the GEANT4 all purpose set of physics processes and interaction models. However, for low energy applications such as medical physics and dose calculations, the low energy electromagnetic processes package is preferred (see http://www.ge.infn.it/geant4/lowE/). The low energy package is an alternative to the standard physics processes that extends the validity range of particle interactions to lower energies (a few hundred $\mathrm{eV}$ to about $1 \mathrm{GeV}$ ). The package includes the photoelectric effect, Compton scattering, Rayleigh scattering, Bremsstrahlung and ionization. Fluorescence from excited atoms is also treated.

Dose calculations obtained with GATE have been compared to other Monte Carlo Codes, namely MCNP and EGSnrc. A simple benchmark, suggested earlier by Rogers et al, and easy to implement in all codes, has been used to compare the performance of all three MC codes. The benchmark describes experiments (one with photons and one with electrons) in which a planar beam in vacuum is impinging on a phantom made of slabs of different materials (water, aluminium, lung, water). The dose is reported along the central axis in the phantom. The 
phantom is a matrix of $61 \times 79 \times 150$ voxels of $5 \times 5 \times 2$ $\mathrm{mm}$ each. For the photon experiment, an 18MV beam calculated from a clinical accelerator model was used and for the electron case, a $20 \mathrm{MeV}$ monoenergetic beam was used.

In the case of GATE, simulations were run on dual $3.2 \mathrm{GHz}$ Xeon processors with 4GB of memory installed. For the photon experiment, $10^{7}$ photons were emitted onto the phantom according to the energy spectrum. For the electron experiment, $10^{6}$ electrons were emitted onto the phantom. With EGSnrc on a dual $2.4 \mathrm{GHz}$ Xeon with $1 \mathrm{~GB}$ of memory, a total of $5 \times 10^{7}$ and $2 \times 10^{7}$ were emitted for the photons and the electrons respectively. Photon splitting as variance reduction technique (VRT) was used during the simulation of the photon beam with EGSnrc. Finally using MCNPX2, run on dual 2GHz processor Mac G5 with $1 \mathrm{~GB}$ of memory, a total of $2 \times 10^{7}$ photons were simulated for the photon beam.

The comparative efficiency of the simulation using the different MC codes was assessed using

$$
\varepsilon=\frac{1}{T \sigma^{2}}
$$

where, $\mathrm{T}$ is the CPU time for the simulation and $\sigma$ is the statistical uncertainty of the quantity of interest ${ }^{22}$.

\subsection{Dosimetry examples with GATE}

Examples for the use of GATE in dosimetry applications already exist for both animal and human models. In terms of small animal imaging GATE dosimetric capabilities have been used to calculate dose in mice from CT protocols and PET FDG studies. A realistic mouse phantom from published work $^{23}$ was used for dose calculations.

For the CT study, the MicroCAT II small animal scanner (ImTek Inc, Knoxville, TN) was modeled and the $\mathrm{x}$-ray energy spectrum was calculated for tube voltages of 50, 70 and $80 \mathrm{kVp}$ with 1,2 and 3 $\mathrm{mm}$ of Aluminium filtering, respectively. The mouse phantom was placed in an acrylic, cylindrical mouse chamber phantom similar to the one used in actuality. A total of $2 \times 10^{9}$ photons were emitted $360^{\circ}$ around the phantom to simulate the procedure. Dose-volume histograms were calculated for each organ using the dose matrix and the phantom matrix for organ identification.

A PET-FDG dose study has also been conducted. Dynamic biodistributions of FDG in an anesthetized $26 \mathrm{~g}$ mouse were obtained after drawing regions of interest on images from a multi time-frame PET study. Discrete time-activity curves were calculated and transposed to the voxellized mouse phantom. Simulations were performed on 10 time intervals of constant FDG distribution, each tracking $10^{8}$ positron histories. Calculated dose was scaled to take into account radioactive decay, time integration and branching ratios.

Radiotherapy and brachytherapy experiments have been also modeled in analytically defined volumes using GATE. In terms of radiotherapy a complete linear accelerator system emitting electrons has been modeled while specific applications in brachytherapy include the use of ${ }^{106} \mathrm{Ru}$ as a beta emitter in ophthalmic applicators $^{24}$.

\section{Results}

\subsection{Dosimetry Benchmark}

Figure 1 shows the normalized (to particle fluence) depth-dose profile for the $18 \mathrm{MV}$ photon experiment considering the MC codes under evaluation. In a similar fashion Figure 2 shows the depth-dose behaviour for the $20 \mathrm{MeV}$ electron experiment. In terms of speed of execution GATE was slower than EGSnrc by a factor of 18 and 3 in the case of photons and electrons respectively. Comparing the speed of execution, it is important to notice the use of different processors for each of the codes. However, we can still account for the larger difference observed with the photon beam as a result of the photon splitting used with EGSnrc. In terms of overall dose uncertainty the results between MCNP and GATE were similar $(2.3 \%$ at a depth of $10 \mathrm{~cm})$, while EGSnrc was better for both electrons and photons by a factor of 3-4. This is due to differences in the methodology used for the calculation of the dose uncertainty by the different codes. In GATE, the uncertainty is calculated on each of the interactions within a given voxel, while in EGSnrc the energy 


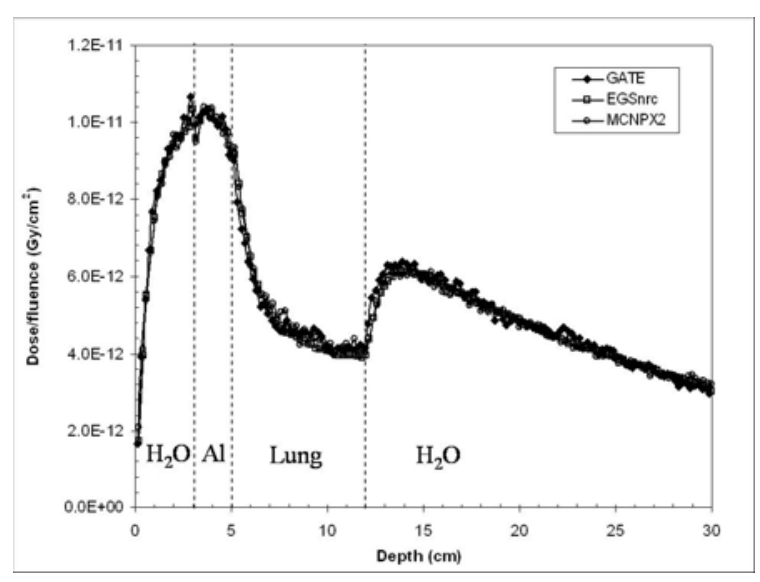

Fig. 1. Depth dose curve using the benchmark and the 18MV photon beam for GATE, EGSnrc and MCNPX2

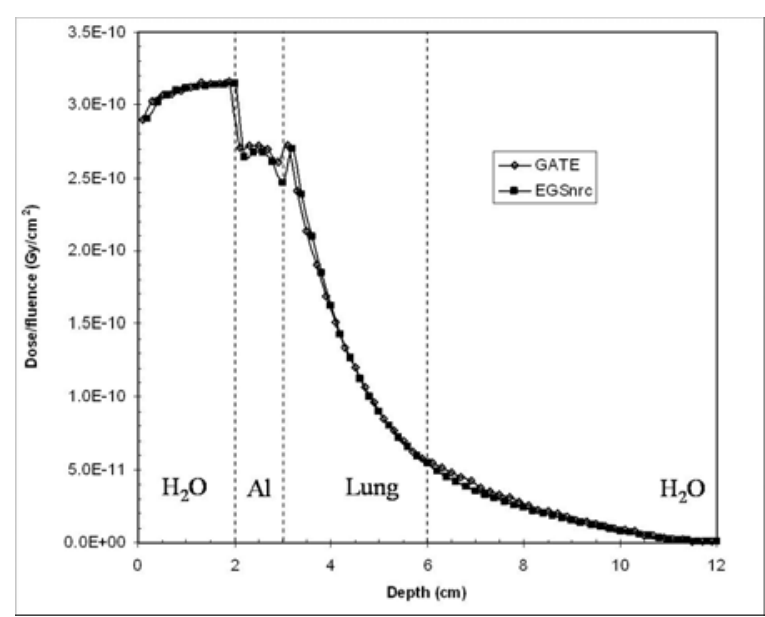

Fig. 2. Depth dose curve using the benchmark and the $20 \mathrm{MeV}$ electron beam for GATE and EGSnrc.

deposited by the different particles in a voxel is firstly summed and the uncertainty is subsequently calculated. Therefore in the case of GATE uncertainty calculations, the way it is currently implemented, particles depositing a small energy have a large effect in dose uncertainty estimation.

\subsection{Dosimetry examples with GATE}

Considering the microCT study results showed that for a $70 \mathrm{kVp} / 2 \mathrm{~mm} \mathrm{Al}$ beam and a protocol calling for 360 exposures of $0.5 \mathrm{~s}$ with a current of $0.5 \mathrm{~mA}$ (total of $90 \mathrm{mAs}$ ), an average dose of $9 \mathrm{cGy}$ is given to the mouse.
In the PET FDG dose study most organs received an average dose of about 1 cGy per injected MBq. Dose to the heart was about $4 \mathrm{cGy} / \mathrm{MBq}$ and the bladder wall received about $50 \mathrm{cGy} / \mathrm{MBq}$, for the unvoided bladder, an about half for a partially voided bladder 90 minutes after injection.

Figure 3 shows a comparison of isodose contours produced using different codes in the case of ${ }^{106} \mathrm{Ru}$ based ophthalmic applicators, demonstrating a good agreement between GATE and measurements with other MC codes. In the case of radiotherapy units a good agreement was seen between extrapolation chamber measurement on the central axis of a water phantom and GATE calculations.

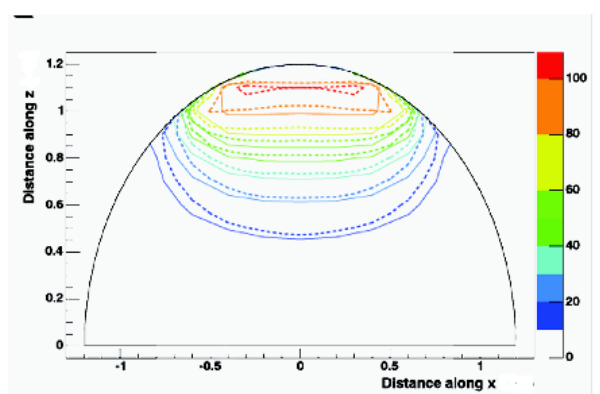

Fig. 3. Comparison of isodose contours for doses in water from a CCA BEBIG applicator between GATE (dashed curves) and Plaque Simulator ${ }^{25}$ analytic calculations (solid curves). Doses normalized to $100 \%$ at $1 \mathrm{~mm}$.

\section{Discussion}

GATE is already in use for dosimetry applications in both animal and human models. However, certain challenges still remain in order to allow GATE as a mainstream MC code for dosimetry applications. This includes deficiencies associated with specific GEANT4 particle tracking modules such as boundary crossing models for electron transport simulations ${ }^{19}$ as well as the multiple scattering algorithm. Future releases by the GEANT4 collaboration will be addressing these problems.

Another issue that can be identified as a significant disadvantage for the use of GATE in dosimetry applications is in terms of simulation efficiency. GATE which is based on GEANT4 suffers from the lack of variance reduction techniques such as those present in dosimetry specific codes such as EGSnrc and MCNP. Different solutions can 
be envisaged in order to address GATE simulation efficiency issues, some of which can be specific for dosimetry applications. One such solution is to intrinsically improve the tracking speed for dosimetry applications by referring to methods elaborately described in the (X)VMC papers ${ }^{4,26-27}$. An analogue implementation of the beam model (as in external radiotherapy) can be designed, based on the nuclear medicine image of the pre-therapy tracer. Such a ray tracing technique, consisting of precalculating the number of primary photon interactions in each $\operatorname{voxel}^{28}$, will drastically improve the simulation efficiency. The challenge in the implementation of this method lies with in the description of the equivalent of the beam model for internal radiotherapy. Simple approximations can be made using monoenergetic photon beams or point sources with a certain energy distribution. Such approximations can be derived using the pre-therapy SPECT/PET tracer study. Energy dependence of this equivalent internal radiotherapy beam model will be binned and material properties will be read from voxelised CT data as already implemented in GATE.

Alternative, efficiency improvements that are currently under evaluation in labs of the openGATE collaboration, can be derived by combining voxels with the same linear attenuation coefficient in voxellised sources reducing the number of interfaces a particle has to "face" before exiting the phantom. Another solution is the parallel implementation of the GEANT4 events generation scheme for a most efficient use of GATE and computer clusters.

GATE developments will be equally considered on the implementation of a variety of dose scoring techniques, including separation of the dose from primary and scattered photons that have been previously developed for brachytherapy dose simulations ${ }^{29}$.

In conclusion, a dose calculation module has been added to GATE allowing dose maps to be derived for complicated voxelised sources. This new module coupled with the flexibility of GATE in modeling complicated detector and phantom geometries may lead to a powerful tool for dosimetry applications. A variety of dose scoring techniques has been previously developed for brachytherapy simulations, and GATE developments in this direction should be also considered. Finally, future work will concentrate on improving the speed of execution, adding larger flexibility in the dose calculation module, and further validation studies in the use of GATE for internal and external dosimetry applications.

\section{References}

[1] S. Jan, G. Santin, D. Strul et al. Phys Med Biol 49 (2004) 4543.

[2] J.F. Briesmeister, Report LA-13709-M, Los Alamos National Laboratory, NM, 2000.

[3] I. Kawrakow and D.W.O. Rogers, NRCC Report PIRS-701, National Research Council of Canada, Ottawa, 2001.

[4] M. Fippel, Med Phys 26, 1466 (1999)

[5] D.W.O Rogers and R Mohan, http://www.irs.inms.nrc.ca/ inms/irs/papers/iccr00/iccr00.html (2000).

[6] I. Buvat and I. Castiglioni, Q J Nucl Med 46 (1), 48, (2002).

[7] D. W. O. Rogers and A.F. Bielajew, in The Dosimetry of Ionizing Radiation, (Academic Press, 1990), Vol. III.

[8] F. Verhaegen and J. Seuntjens, Phys Med Biol 48 (21), R107 (2003).

[9] C. M. Ma and S. B. Jiang, Phys Med Biol 44 (12), R157 (1999).

[10] Kawrakow I, Med. Phys., 485 (2000).

[11] Kawrakow I, Med. Phys. 27, 499 (2000).

[12] I. Kawrakow, D. W. Rogers, and B. R. Walters, Med Phys 31 (10), 2883 (2004).

[13] B. R. Walters, I. Kawrakow, and D. W. Rogers, Med Phys 29 (12), 2745 (2002).

[14] O. Chibani, X.A. Li, Med Phys 29 (5), 835 (2002)

[15] F. Difilippo, L. Papiez, V. Moskvin, et al, Med Phys 30 (10), 2849 (2003).

[16] P. H. McGinley, Shielding Techniques for Radiation Oncology Facilities, (Medical Physics Publishing, 2002).

[17] J. F. Carrier, L. Archambault, L. Beaulieu, and R. Roy, Med Phys 31 (3), 484 (2004).

[18] E. Poon and F. Verhaegen, Med Phys 32 (6), 1696 (2005).

[19] P. Rodrigues, A. Trindade, L. Peralta, et al, Appl Radiat Isot 61 (6), 1451 (2004)

[20] E. Poon, J. Seuntjens, F. Verhaegen, Phys Med Biol 50, 681, (2005).

[21] Agostinelli S et al, Nucl Instr Meth A506, 250, (2003)

[22] I. Kawrakow, Med Phys 32(7), 2320 (2005).

[23] W.P. Segars, B.M. Tsui, E.C. Frey, et al, Mol Imag Biol 6(3), 149 (2004)

[24] http://www.bebig.de

[25] M.A.Astrahan, Med Phys 30(6), 1219, 2003

[26] M. Fippel, I. Kawrakow, K. Friedrich, Phys Med Biol 42, 501 (1997)

[27] M. Fippel, I. Kawrakow, Phys Med Biol 45, 2163 (2000)

[28] L. Wang, S. Chui, M. Lovelock, Med Phys 25, 867 (2000)

[29] A.S. Kirov, J.F. Williamson, Phys Med Biol 42 (11), 2119 (1997). 